MIFP-09-14

NSF-KITP-09-30

\title{
Supersymmetry breaking, heterotic strings and fluxes
}

\author{
Katrin Becker, Chris Bertinato, Yu-Chieh Chung and Guangyu Guo \\ Department of Physics, Texas A\&M University, \\ College Station, TX 79843, USA
}

\begin{abstract}
In this paper we consider compactifications of heterotic strings in the presence of background flux. The background metric is a $\mathrm{T}^{2}$ fibration over a K3 base times fourdimensional Minkowski space. Depending on the choice of three-form flux different amounts of supersymmetry are preserved $(\mathrm{N}=2,1,0)$. For supersymmetric solutions unbroken space-time supersymmetry determines all background fields except one scalar function which is related to the dilaton. The heterotic Bianchi identity gives rise to a differential equation for the dilaton which we discuss in detail for solutions preserving an $\mathrm{N}=2$ supersymmetry. In this case the differential equation is of Laplace type and as a result the solvability is guaranteed.
\end{abstract}

April 2009

Email: kbecker, cbertinato, ycchung, guangyu@physics.tamu.edu 


\section{Introduction}

In this paper we study different aspects of string theory compactifications in the presence of background flux. Our main focus is the heterotic string compactified to four dimensions with background NS three-form $\mathcal{H}$. Compactifications of heterotic strings are interesting, both from the phenomenological and from the mathematical point of view. In general, unbroken supersymmetry requires the background in a compactitification of the heterotic string to be a complex space with an hermitian metric but does not require the space to be Kähler. If the background is non-Kähler the NS threeform is non-vanishing and the deviation from "Kählerity" is measured by the flux. On these spaces there exists a globally defined two-form $J$ which is no longer closed. Its derivative is rather related to the flux by [1] [2]

$$
\mathcal{H}=i(\partial-\bar{\partial}) J .
$$

While the Kähler case, like Calabi-Yau and torus compactifications, have been intensively studied much less is known about the generic case in which the compactification manifold is no longer Kähler. From the mathematical point of view algebraic geometry techniques are still missing even though some progress has been made in describing these spaces with an explicit metric [3].

We will consider torsional heterotic backgrounds which are a $\mathrm{T}^{2}$ fibered over a K3 base. Depending on the choice of flux different amounts of four-dimensional supersymmetry are preserved. While solutions preserving an $\mathrm{N}=2,1$ supersymmetry have been discussed before in the literature, starting with ref. [4] (see in particular [5] [6]), the supersymmetry breaking solutions are new. We explicitly check that the backgrounds solve the equations of motion. For solutions preserving an $\mathrm{N}=1,0$ supersymmetry we check this at the SUGRA level. While for solutions preserving an $\mathrm{N}=2$ supersymmetry we show how to solve the equations of motion including the first $\alpha^{\prime}$ correction. The spinor equations determine the background except one scalar function related to the dilaton. The Bianchi identity for $\mathcal{H}$ gives rise to a differential equation for this scalar function which is of Laplace type.

We start by discussing, and mostly reviewing, flux compactifications of type IIB string theory on $\mathrm{K} 3 \times \mathrm{T}^{2}$ orientifolds (see for example refs. [4] [7] [8]). Depending on the choice of flux the solutions preserve an $\mathrm{N}=2,1,0$ supersymmetry in four dimensions. The backgrounds solve the equations of motion and in the supersymmetric case the spinor equations. We check this to the leading order in $\alpha^{\prime}$, i.e. in the SUGRA approximation. 
This type IIB background is dual to an heterotic SUGRA background with nonvanishing $\mathcal{H}$-flux and non-trivial dilaton. The duality chain has been described explicitly in ref. [4] based on earlier work by Sen [9] [10]. The heterotic background metric is a $\mathrm{T}^{2}$ fibred over a $\mathrm{K} 3$ base. We are interested in analyzing $\alpha^{\prime}$ corrections to the heterotic SUGRA background. Even though the heterotic and type IIB backgrounds are related by duality we will not use duality to obtain the $\alpha^{\prime}$ corrected heterotic background. Rather we will follow a different route and construct the $\alpha^{\prime}$ corrected background directly on the heterotic side. The reason being that the present knowledge about the relevant interactions on the world-volume of $\mathrm{D} p$-branes and O-planes is insufficient. So for example, the anomalous couplings described in refs. [11] [12] [13] [14] [15] [16] [17] are not compatible with T-duality and additional dependence on NS-NS and R-R fields are required to obtain world-volume actions compatible with T-duality. On the heterotic side, on the other hand, the action and supersymmetry transformations are known to all relevant orders. The low-energy effective action of the heterotic string to $O\left(\alpha^{\prime 3}\right)$ has been constructed by Bergshoeff and de Roo by supersymmetrizing the Chern-Simons term [21]. Our goal is to construct the background which solves the $\alpha^{\prime}$ corrected equations of motion.

The duality between type IIB and heterotic flux backgrounds can be explicitly checked at the level of SUGRA but beyond leading order the duality map makes predictions about higher derivative corrections to the world-volume action describing $\mathrm{D} p$-branes in type IIB theories. Thus, duality, while not being fundamental to construct heterotic flux backgrounds, can be used as a tool to learn about D $p$-brane effective actions. Furthermore duality to type IIB allows us to speculate about the effect of fluxes on four-dimensional phenomenology arising from heterotic strings in generic backgrounds. So for example, the non-trivial heterotic dilaton profile plays a role similar to the warp factor in type IIB compactifications and in regions with strong warping could give rise to large hierarchies.

In section 2 we present the type IIB flux backgrounds. To set up our notation we review the low-energy effective 'action' in section 2.1 and derive the equations of motion of type IIB SUGRA in section 2.2. In section 2.3 we present the background which solves the equations of motion of type IIB SUGRA and check the amount of four-dimensional supersymmetry preserved by the different backgrounds in section 2.4. Taking the type IIB background as a starting point we proceed in section 3 to construct the heterotic flux background. To set up the notation we review in section 3.1 
the heterotic effective action to $O\left(\alpha^{\prime}\right)$ and in section 3.2 we derive the corresponding equations of motion. In section 3.3 we present the background and show that it solves the SUGRA equations of motion. In section 4 we discuss the $\alpha^{\prime}$ corrected background. We start by presenting explicit results for $\operatorname{tr}(R \wedge R)$ which are needed to solve the Bianchi identity and Einstein equation. In section 4.1 we review the proof that $\operatorname{tr}(R \wedge R)$ is a four-form of type $(2,2)$ to leading order in $\alpha^{\prime}$, a condition which is needed for the solvability of the Bianchi identity. In section 4.3, focusing on solutions with $\mathrm{N}=2$ supersymmetry, we show how to construct the background which solves the $\alpha^{\prime}$ corrected Bianchi identity and supersymmetry transformations.

\section{Type IIB SUGRA background}

In this section we review type IIB flux backgrounds in which the space-time metric is a warped product of flat 4d Minkowski space and a $\mathrm{K} 3 \times \mathrm{T}^{2}$ orientifold (see refs. [4] [7] [8] [23] [24]). To set up the notation we start summarizing our conventions for the type IIB SUGRA 'action' together with the corresponding equations of motion. Then we summarize the solutions preserving different amounts of four-dimensional supersymmetry. The analysis is done at the level of SUGRA, i.e. without taking actions describing brane sources into account.

\subsection{The action}

The bosonic part of the type IIB supergravity 'action' in the 10d string frame is

$$
S=S_{\mathrm{NS}}+S_{\mathrm{R}}+S_{\mathrm{CS}}
$$

Here $S_{\mathrm{NS}}$ is

$$
S_{\mathrm{NS}}=\frac{1}{2 \kappa^{2}} \int d^{10} x \sqrt{-g} e^{-2 \phi_{\mathrm{B}}}\left[R+4\left(\partial \phi_{\mathrm{B}}\right)^{2}-\frac{1}{2}\left|H_{3}\right|^{2}\right],
$$

while the parts of the action describing the massless R-R sector fields are given by

$$
\begin{gathered}
S_{\mathrm{R}}=-\frac{1}{4 \kappa^{2}} \int d^{10} x \sqrt{-g}\left(\left|F_{1}\right|^{2}+\left|\widetilde{F}_{3}\right|^{2}+\frac{1}{2}\left|\widetilde{F}_{5}\right|^{2}\right), \\
S_{\mathrm{CS}}=\frac{1}{4 \kappa^{2}} \int C_{4} \wedge H_{3} \wedge F_{3} .
\end{gathered}
$$

In these formulas $F_{n+1}=d C_{n}, H_{3}=d B_{2}$ and

$$
\widetilde{F}_{3}=F_{3}-C_{0} H_{3}
$$




$$
\widetilde{F}_{5}=F_{5}-\frac{1}{2} C_{2} \wedge H_{3}+\frac{1}{2} B_{2} \wedge F_{3}
$$

\subsection{Equations of motion}

The equations of motion are as follows

$$
\begin{aligned}
& d \star F_{1}=\star \tilde{F}_{3} \wedge H_{3}, \\
& d \star \tilde{F}_{3}=\tilde{F}_{5} \wedge H_{3}, \\
& d \star \tilde{F}_{5}=-F_{3} \wedge H_{3},
\end{aligned}
$$

from the R-R fields, and

$$
\begin{aligned}
& R-4\left(\partial \phi_{\mathrm{B}}\right)^{2}+4 \nabla^{2} \phi_{\mathrm{B}}-\frac{1}{2}\left|H_{3}\right|^{2}=0, \\
& d\left(e^{-2 \phi_{\mathrm{B}}} \star H_{3}\right)=F_{1} \wedge \star \tilde{F}_{3}-\tilde{F}_{5} \wedge \tilde{F}_{3},
\end{aligned}
$$

in the NS-NS sector. The variation of the action with respect to the metric leads to

$$
G_{M N}+e^{2 \phi_{\mathrm{B}}}\left(g_{M N} \nabla^{2} e^{-2 \phi_{\mathrm{B}}}-\nabla_{M} \nabla_{N} e^{-2 \phi_{\mathrm{B}}}\right)=-\frac{2 \kappa^{2}}{\sqrt{-g}} \frac{\delta S_{\text {tensor }}}{\delta g^{M N}} e^{2 \phi_{\mathrm{B}}},
$$

where $G_{M N}$ is the Einstein tensor and $S_{\text {tensor }}$ is the action for all the tensor fields including the dilaton. The left hand side arises from the variation of the Einstein-Hilbert action with the dilaton contribution arising from the non-canonically normalized curvature term.

Moreover, the tensor fields satisfy the Bianchi identities

$$
\begin{aligned}
& d H_{3}=0, \\
& d F_{1}=0, \\
& d \tilde{F}_{3}=H_{3} \wedge F_{1}, \\
& d \tilde{F}_{5}=H_{3} \wedge F_{3} .
\end{aligned}
$$

\subsection{The SUGRA background}

We are interested in a solution of the $10 \mathrm{~d}$ equations of motion in which the spacetime contains four non-compact dimensions and six compact dimension. We require maximal symmetry in the non-compact dimensions which means all tensor fields except $F_{5}$ have components along the internal directions only, while $F_{5}$ is required to take the form

$$
\tilde{F}_{5}=(1+\star) d \alpha(y) \wedge d x^{0} \wedge d x^{1} \wedge d x^{2} \wedge d x^{3}
$$


where $x, y$ denote the $4 \mathrm{~d}$ and $6 \mathrm{~d}$ coordinates respectively.

Moreover, we would like to consider a background which arises as the orientifold limit of a flux background of M-theory compactified on $\mathrm{K} 3 \times \mathrm{K} 3$. In this case the RR axion vanishes and the type IIB dilaton $\phi_{\mathrm{B}}$ is constant. The space-time metric is of the form

$$
d s^{2}=e^{2 A(y)+\phi_{\mathrm{B}} / 2} \eta_{\mu \nu} d x^{\mu} d x^{\nu}+e^{-2 A(y)+\phi_{\mathrm{B}} / 2}\left(g_{i j} d y^{i} d y^{j}+d w_{1}^{2}+d w_{2}^{2}\right)
$$

where $g_{i j}$ is the metric of K3 and the factor involving the dilaton arises since this is the metric in the $10 \mathrm{~d}$ string frame and $e^{-2 A(y)}$ is the warp factor depending on the coordinates of the internal space only. The function $\alpha$ in (2.11) is related to the warp factor according to

$$
\alpha(y)=e^{4 A(y)} .
$$

The complex three-form $G_{3}=\tilde{F}_{3}-i e^{-\phi_{\mathrm{B}}} H_{3}$ is imaginary self-dual in the internal dimensions, i.e.

$$
\star G_{3}=i G_{3} .
$$

Moreover, the warp factor satisfies the Poisson equation

$$
\nabla^{2} e^{-4 A(y)}+e^{-\phi_{\mathrm{B}}}\left|H_{3}\right|^{2}=0 \text {. }
$$

Away from the orientifold points this is a solution of the equations of motion as can be explicitly verified.

Note that the three-form tensor fields $H_{3}$ and $\tilde{F}_{3}$ are harmonic forms on the internal part of the space (2.12). It turns out that the Hodge numbers of K3 are

$$
\begin{aligned}
& h^{0,0} \quad 1 \\
& h^{1,0} \quad h^{0,1} \quad 0 \quad 0 \\
& h^{2,0} \quad h^{1,1} \quad h^{0,2}=1 \quad 20 \quad 1 \\
& h^{2,1} h^{1,2} \quad 0 \quad 0 \\
& h^{2,2} \quad 1
\end{aligned}
$$

and in particular there are no harmonic one-forms or three-forms on K3. As a result $H_{3}$ and $\tilde{F}_{3}$ have to be the product of harmonic two-forms on $\mathrm{K} 3$, which we will denote by $\left(h_{3}\right)_{i}$ and $\left(\tilde{f}_{3}\right)_{i}$ and a one-form in the fiber directions, $d w^{i}$, i.e.

$$
H_{3}=\left(h_{3}\right)_{i} \wedge d w^{i} \quad \text { and } \quad \tilde{F}_{3}=\left(\tilde{f}_{3}\right)_{i} \wedge d w^{i}, \quad i=1,2,
$$


where $w_{i} \sim w_{i}+1$ and

$$
\left(\tilde{f}_{3}\right)_{i},\left(h_{3}\right)_{i} \in H^{2}(K 3, \mathbb{Z}) .
$$

Moreover, the condition that $G_{3}$ is imaginary self-dual requires the complex three-form to be

$$
G_{3}=g_{+} \wedge d \bar{w}+g_{-} \wedge d w
$$

where

$$
d w=d w_{1}+i d w_{2}
$$

and $g_{ \pm}$can be expanded in (anti)-self dual harmonic two-forms on K3

$$
g_{+} \in H^{2,0}(K 3) \oplus H^{0,2}(K 3) \oplus H_{+}^{1,1}(K 3) \quad \text { and } \quad g_{-} \in H_{-}^{1,1}(K 3) .
$$

There are 3 self-dual two-forms and 19 anti-self dual two-forms which are of type $(1,1)$ and primitive. In the following we will see that the different solutions of the equations of motion preserve different amounts of supersymmetry. In particular, the amount of unbroken supersymmetry will depend on the choices of two-forms on K3.

\subsection{Supersymmetry}

Let us represent the dilatino and gravitino fields by Weyl spinors $\lambda$ and $\Psi_{\mu}$, respectively. Similarly, the infinitesimal supersymmetry parameter is represented by a Weyl spinor $\varepsilon$. The supersymmetry transformations of the fermi fields of type IIB supergravity (to leading order in fermi fields) are

$$
\delta \lambda=\frac{1}{2}\left(\not \partial \phi_{\mathrm{B}}-i e^{\phi_{\mathrm{B}}} \not \partial C_{0}\right) \varepsilon+\frac{1}{4}\left(i e^{\phi_{\mathrm{B}}} \widetilde{H}_{3}-H_{3}\right) \varepsilon^{\star},
$$

and

$$
\delta \Psi_{M}=\left(\nabla_{M}+\frac{i}{8} e^{\phi_{\mathrm{B}}} \mathscr{H}_{1} \Gamma_{M}+\frac{i}{16} e^{\phi_{\mathrm{B}}} \widetilde{\not}_{5} \Gamma_{M}\right) \varepsilon-\frac{1}{8}\left(2\left(H_{3}\right)_{M}+i e^{\phi_{\mathrm{B}}} \widetilde{H}_{3} \Gamma_{M}\right) \varepsilon^{\star} .
$$

Upon reducing to $4 \mathrm{~d}$ the Lorentz algebra decomposes according to $S O(9,1) \rightarrow$ $S O(3,1) \times S O(6)$. The Weyl spinor $\varepsilon$ then decomposes as

$$
16 \rightarrow(2,4)+\left(2^{\prime}, 4^{\prime}\right)
$$

Under the further decomposition $S O(6) \rightarrow S O(4) \times S O(2)$

$$
\begin{gathered}
4 \rightarrow(2,1)+\left(2^{\prime}, 1^{\prime}\right) \\
4^{\prime} \rightarrow\left(2,1^{\prime}\right)+\left(2^{\prime}, 1\right)
\end{gathered}
$$


The holonomy of $\mathrm{K} 3$ is $S U(2)$ and under the reduction $S O(4) \rightarrow S U(2)$

$$
\begin{aligned}
& 2 \rightarrow 1+1 \\
& 2^{\prime} \rightarrow 2 .
\end{aligned}
$$

This means that either $\mathbf{4}$ or $\mathbf{4}^{\prime}$ of $S O(6)$ gives rise to two $S U(2)$ singlets leading to an $\mathrm{N}=4$ supersymmetry in $4 \mathrm{~d}$.

Next we analyze the constraints imposed by the orientifold projection $\mathbb{Z}_{2}=$ $\Omega(-1)^{F_{L}} \mathcal{I}$. Writing $\varepsilon=\varepsilon_{1}+i \varepsilon_{2}$ the different parity transformations act according to

$$
\varepsilon=\varepsilon_{1}+i \varepsilon_{2} \stackrel{\Omega}{\rightarrow} \varepsilon_{2}+i \varepsilon_{1} \stackrel{(-1)^{F_{L}}}{\rightarrow}-\varepsilon_{2}+i \varepsilon_{1} \stackrel{\mathcal{I}}{\rightarrow} i \Gamma_{\star}\left(-\varepsilon_{2}+i \varepsilon_{1}\right),
$$

where $\Gamma_{\star}$ is the chirality operator of $S O(2)$. Combining these operations and requiring the spinor to be left invariant by the orientifold action imposes

$$
\varepsilon=-\Gamma_{\star} \varepsilon
$$

Before we proceed, lets determine how the spinor projection relates to the one in the type I string. After two T-dualities on torus, the left moving spinor $\varepsilon_{1}$ is unaffected, however the right moving spinor $\varepsilon_{2}$, transforms as

$$
\varepsilon_{2} \rightarrow \Gamma^{8} \Gamma^{9} \varepsilon_{2}
$$

from which we get the transformation of Eq.(2.28),

$$
\left(1+\Gamma_{\star}\right)\left(\varepsilon_{1}-\varepsilon_{2}\right)=0
$$

Because the gamma matrix $\Gamma_{\star}$ is pure imaginary in our representation, this condition leads to $\varepsilon_{1}=\varepsilon_{2}$, the spinor that survives the world sheet projection of type IIB string, i.e. type I string. This is an alternative way to see how type I string emerges after performing T-dualities of type IIB orientifold.

Eqn.(2.28) means that spinor has a definite chirality on the torus, which we choose to be 1 in eqn. (2.25), while $\mathbf{1}^{\prime}$ is projected out. As a result the $S U(2)$ singlets which are not projected out by the orientifold arise from the 4 in eqn. (2.25). The orientifold breaks the $4 \mathrm{~d}$ supersymmetry from $\mathrm{N}=4$ to $\mathrm{N}=2$. Moreover, the two $4 \mathrm{~d}$ spinors are in the 2 of $S O(3,1)$ so have the same chirality. We denote the resulting spinors by $\eta_{i}$, and by an $S O(4)$ transformation we can choose them to satisfy

$$
\Gamma_{i} \eta_{1}=\Gamma_{w} \eta_{1}=0 \quad \text { and } \quad \Gamma_{\bar{i}} \eta_{2}=\Gamma_{w} \eta_{2}=0
$$


where $\left(y^{i}, y^{\bar{i}}\right)$ and $(w, \bar{w})$ are complex coordinates on K3 and the torus respectively.

Using these supersymmetry transformations the unbroken supersymmetries are those that satisfy $\delta$ (fermi) $=0$. Evaluated in the background metric (2.12), using the relation between the warp factor $A(y)$ and $\alpha(y)$ and the fact that the spinors have definite $4 \mathrm{~d}$ chirality the supersymmetry conditions become

$$
\nabla_{i}\left(e^{-A / 2} \varepsilon\right)=0
$$

which is satisfied with a spinor proportional to the covariantly constant spinors on $\mathrm{K} 3 \times \mathrm{T}^{2}$ and

$$
\mathbf{G}_{m} \varepsilon^{\star}=0 \quad \text { and } \quad \mathbf{G} \varepsilon=\mathbf{0} .
$$

Next we solve the constraints (2.33) and we will check that depending on the choice of flux different amounts of supersymmetry are preserved. Lets analyze the amount of unbroken supersymmetry

$\triangleright$ if $G=g_{-} \wedge d w$, then

$$
G_{w i \bar{j}} \Gamma^{i \bar{j}} \eta_{k}^{\star}=G_{i w \bar{j}} \Gamma^{w \bar{j}} \eta_{k}^{\star}=G_{\overline{j i w}} \Gamma^{i w} \eta_{k}^{\star}=G_{w i \bar{j}} \Gamma^{w i \bar{j}} \eta_{k}=0
$$

for $k=1,2$. This is solved by requiring $G$ to be primitive with respect to the base, i.e.

$$
G_{w i j} g^{i \bar{j}}=0
$$

while both spinors $\eta_{k}$ for $k=1,2$ are non-vanishing. Since $g_{-}$are expanded in a basis of anti-self dual $(1,1)$ forms eqn. (2.35) is always satisfied. This leads to an $\mathrm{N}=2$ supersymmetry in $4 \mathrm{~d}$.

$\triangleright$ if $G=g_{+}^{2,0} \wedge d \bar{w}$, eqn. (2.33) requires

$$
G_{\bar{w} i j} \Gamma^{i j} \eta_{2}^{\star}=0
$$

which is solved by $\eta_{2}=0$, while the conditions on $\eta_{1}$ are

$$
G_{\bar{w} i j} \Gamma^{i j} \eta_{1}^{\star}=G_{i \bar{w} j} \Gamma^{\bar{w} j} \eta_{1}^{\star}=G_{i j \bar{w}} \Gamma^{i j \bar{w}} \eta_{1} .
$$

These conditions are always satisfied which implies that the $4 \mathrm{~d}$ supersymmetry arising from $\eta_{1}$ is unbroken. This flux configuration leads to an $\mathrm{N}=1$ supersymmetry in $4 \mathrm{~d}$. 
$\triangleright$ if $G=g_{+}^{0,2} \wedge d \bar{w}$, eqn. (2.33) requires

$$
G_{\bar{w} \bar{i} \bar{j}} \Gamma^{\bar{i} \bar{j}} \eta_{k}^{\star}=G_{\bar{i} \bar{w} \bar{j}} \Gamma^{\bar{w} \bar{j}} \eta_{k}^{\star}=G_{\bar{j} \bar{i} \bar{w}} \Gamma^{\bar{i} \bar{w}} \eta_{k}^{\star}=G_{\bar{w} \bar{i} \bar{j}} \Gamma^{\bar{w} \bar{i} \bar{j}} \eta_{k}=0
$$

for $k=1,2$. These conditions are solved by requiring $\eta_{1}=0$ while $\eta_{2} \neq 0$ and as a result there is an $\mathrm{N}=1$ ' unbroken supersymmetry in $4 \mathrm{~d}$. We label this supersymmetry with $\mathrm{N}=1$ ' since it preserves a different subgroup of the supersymmetry than the $G_{\bar{w} i j}$ component.

$\triangleright$ if $G=g_{+}^{1,1} \wedge d \bar{w}$, eqn. (2.33) requires $\eta_{1}=\eta_{2}=0$ and supersymmetry is completely broken.

\section{$3 \quad$ Heterotic SUGRA background}

In this section we analyze the heterotic flux backgrounds. To set up the notation we review the heterotic low-energy effective action to $O\left(\alpha^{\prime 2}\right)$ in section 3.1. In section 3.2 we summarize the equations of motion. In section 3.3 we present the backgrounds solving the SUGRA equations to leading order in $\alpha^{\prime}$. In section 3.4 we analyze the amount of unbroken four-dimensional supersymmetry. This section is confined to solutions solving the SUGRA equations to leading order in $\alpha^{\prime}$ and the corrected background is discussed in section 4.

\subsection{The action}

The bosonic part of the heterotic supergravity action to $O\left(\alpha^{\prime 2}\right)$ in the $10 \mathrm{~d}$ string frame is [18] [19] [20] 21] 22]

$$
S_{h e t}=\frac{1}{2 \kappa^{2}} \int d^{10} x \sqrt{-g} e^{-2 \phi_{\mathrm{h}}}\left[R+4\left(\partial \phi_{\mathrm{h}}\right)^{2}-\frac{1}{2}|\mathcal{H}|^{2}-\frac{\alpha^{\prime}}{4} \operatorname{tr}\left(\mathcal{F}^{2}-R_{+}^{2}\right)\right],
$$

where

$$
\mathcal{H}=d \mathcal{B}+\frac{\alpha^{\prime}}{4} \omega_{3}
$$

is the NS three-form and $\mathcal{F}=d \mathcal{A}+\mathcal{A} \wedge \mathcal{A}$ is the gauge field strength. Moreover, $\omega_{3}=\omega_{\mathrm{L}}-\omega_{\mathrm{YM}}$ is given in terms of the Lorentz and Yang-Mills Chern-Simons threeforms

$$
\omega_{\mathrm{L}}=\operatorname{tr}\left(\Omega_{+} \wedge d \Omega_{+}+\frac{2}{3} \Omega_{+} \wedge \Omega_{+} \wedge \Omega_{+}\right) \quad \text { and } \quad \omega_{\mathrm{YM}}=\operatorname{tr}\left(\mathcal{A} \wedge d \mathcal{A}+\frac{2}{3} \mathcal{A} \wedge \mathcal{A} \wedge \mathcal{A}\right) .
$$


The contribution to the action which is quadratic in the Riemann tensor is

$$
\operatorname{tr} R_{+}^{2}=\frac{1}{2} R_{M N A B}\left(\Omega_{+}\right) R^{M N A B}\left(\Omega_{+}\right),
$$

while the quadratic term in $\mathcal{F}$ is the standard gauge field kinetic term. Note that the Einstein-Hilbert action is formulated in terms of the spin connection while the quadratic term in the Riemann tensor is expressed in terms of a connection involving the NS three-form which explicitly is defined by

$$
\Omega_{ \pm M}^{A B}=\Omega^{A B}{ }_{M} \pm \frac{1}{2} \mathcal{H}^{A B}{ }_{M} .
$$

Also, we will follow ref. 21] according to which the action involves the $\Omega_{+}$connection while the supersymmetry transformations involve the $\Omega_{-}$connection. The supersymmetry tranformations will be described in more detail below.

\subsection{Equations of motion}

The equations of motion arising from the action presented in the previous section are

$\triangleright$ for the dilaton

$$
R-4\left(\nabla \phi_{\mathrm{h}}\right)^{2}+4 \nabla^{2} \phi_{\mathrm{h}}-\frac{1}{2}|\mathcal{H}|^{2}-\frac{\alpha^{\prime}}{4} \operatorname{tr}\left(\mathcal{F}^{2}-R_{+}^{2}\right)=0,
$$

$\triangleright$ for $\mathcal{B}$

$$
d\left(e^{-2 \phi_{\mathrm{h}}} \star_{10} \mathcal{H}\right)=0,
$$

$\triangleright$ for the metric

$$
\begin{aligned}
& R_{M N}+2 \nabla_{M} \nabla_{N} \phi_{\mathrm{h}}-\frac{1}{4} \mathcal{H}_{M P Q} \mathcal{H}_{N}{ }^{P Q}+ \\
& \frac{\alpha^{\prime}}{4}\left[R_{M P Q R}\left(\Omega_{+}\right) R_{N}{ }^{P Q R}\left(\Omega_{+}\right)-\mathcal{F}_{M P} \mathcal{F}_{N}{ }^{P}\right]=0,
\end{aligned}
$$

$\triangleright$ for the Yang-Mills field

$$
e^{2 \phi_{\mathrm{h}}} d\left(e^{-2 \phi_{\mathrm{h}}} \star_{10} \mathcal{F}\right)+\mathcal{A} \wedge \star_{10} \mathcal{F}-\star_{10} \mathcal{F} \wedge \mathcal{A}+\mathcal{F} \wedge \star_{10} \mathcal{H}=0 .
$$

The Bianchi identities are

$$
d \mathcal{H}=\frac{\alpha^{\prime}}{4}\left[\operatorname{tr}\left(R_{+} \wedge R_{+}\right)-\operatorname{tr}(\mathcal{F} \wedge \mathcal{F})\right] \quad \text { and } \quad d \mathcal{F}+[\mathcal{A}, \mathcal{F}]=0 .
$$




\subsection{The SUGRA background}

In the following, we present the background that solves the SUGRA equations of motion to leading order in $\alpha^{\prime}$ (see ref. (4 5 [6] for supersymmetric backgrounds). As we will see non-trivial solutions of the Bianchi identity exist only for non-compact backgrounds. This conclusion is modified once $\alpha^{\prime}$ corrections are taken into account.

The background metric is

$$
d s_{\text {het }}^{2}=\eta_{\mu \nu} d x^{\mu} d x^{\nu}+e^{-4 A(y)} g_{i j} d y^{i} d y^{j}+E_{w_{1}} E_{w_{1}}+E_{w_{2}} E_{w_{2}},
$$

where

$$
E_{w_{1}}=d w_{1}+B_{i w_{1}} d y^{i} \quad \text { and } \quad E_{w_{2}}=d w_{2}+B_{i w_{2}} d y^{i},
$$

and $B_{w_{k}}=B_{i w_{k}} d y^{i}$, for $k=1,2$ are one-forms on the base. These one-forms are constrained by the condition that

$$
H_{w_{1}}=d B_{j w_{1}} d y^{j} \quad \text { and } \quad H_{w_{2}}=d B_{j w_{2}} d y^{j},
$$

are harmonic non-trivial two-forms on K3. Note that $E_{w_{k}}$ have to be globally defined since otherwise the metric is not be globally defined. As a result on the $6 \mathrm{~d}$ space $H_{w_{k}}=d E_{w_{k}}$ become exact even though these forms are non-trivial on K3. We will expand $H_{w_{k}}$ in harmonic non-trivial two-forms on K3. Depending on the choice of flux different amounts of $4 \mathrm{~d}$ supersymmetry will preserved as we will see in the next section.

The three-form is

$$
\mathcal{H}=e^{2 \phi_{\mathrm{h}}} \star_{6} d\left(e^{-2 \phi_{\mathrm{h}}} E^{w_{1}} \wedge E^{w_{2}}\right)=\star_{b} d e^{-4 A(y)}-\star_{b} H_{w_{1}} \wedge E^{w_{1}}-\star_{b} H_{w_{2}} \wedge E^{w_{2}},
$$

where $\star_{6}$ denotes the Hodge dual with respect to the $6 \mathrm{~d}$ internal space and $\star_{b}$ denotes the Hodge dual with respect to the unwarped base.

The dilaton is given by

$$
\phi_{\mathrm{h}}=-2 A(y) .
$$

The Yang-Mills field is assumed to be a two-form on K3 only and to satisfy the hermitian Yang-Mills equations, i.e.

$$
\mathcal{F}_{i \bar{j}} J^{i \bar{j}}=0 \quad \text { and } \quad \mathcal{F}_{i j}=\mathcal{F}_{\bar{i} \bar{j}}=0 .
$$

Here $J$ is the Kähler form of K3. 
Moreover, $A(y)$ is a scalar function depending on the coordinates of the base only. To leading order it is required to solve the differential equation

$$
\nabla^{2} e^{-4 A(y)}+\left|H_{w_{1}}\right|^{2}+\left|H_{w_{2}}\right|^{2}=0
$$

Next we show that this background satisfies the equations of motion to leading order in $\alpha^{\prime}$. The equation of motion of $\mathcal{B}$ is satisfied since (3.14) implies

$$
\star_{10} \mathcal{H}=-e^{2 \phi_{\mathrm{h}}} d\left(e^{-2 \phi_{\mathrm{h}}} E^{w_{1}} \wedge E^{w_{2}}\right) \wedge d x^{0123} .
$$

The equation of motion for the metric has several components

$$
(\mu, \nu),(i, j),\left(w_{1}, i\right),\left(w_{2}, i\right),\left(w_{1}, w_{2}\right) .
$$

The $(i, j)$ component, with two indices on $\mathrm{K} 3$, is satisfied assuming $A(y)$ solves (3.17). Moreover, it is easy to see that all other components vanish to this order in $\alpha^{\prime}$.

Next we consider the dilaton equation of motion. Using the metric (3.11) to compute the scalar curvature $R$, the dilaton equation of motion is solved assuming $A(y)$ solves eqn. (3.17). On the other hand the Bianchi identity leads to

$$
d \mathcal{H}=-\left(\nabla^{2} e^{-4 A(y)}+\left|H_{w_{1}}\right|^{2}+\left|H_{w_{2}}\right|^{2}\right) \star_{b} 1=0,
$$

which again is solved after imposing eqn. (3.17)

Note that eqn. (3.17) only has non-trivial solutions if the internal space is noncompact. Below we will describe in detail how to construct compact solutions by going beyond the leading order in $\alpha^{\prime}$.

\subsection{Supersymmetry}

Next let us analyze the supersymmetry of the solutions of the equation of motion. The supersymmetry transformations leaving the 10d heterotic string frame effective action invariant are

$$
\begin{aligned}
& \delta \Psi_{M}=\nabla_{M} \varepsilon-\frac{1}{4} \mathcal{H}_{M} \varepsilon, \\
& \delta \lambda=\not \partial \phi_{h} \varepsilon-\frac{1}{2} \mathcal{H} \varepsilon, \\
& \delta \chi=2 \mathcal{F} \varepsilon,
\end{aligned}
$$

where $\Psi_{M}$ is the gravitino, $\lambda$ the dilatino and $\chi$ the gaugino. All spinors are MajoranaWeyl. The covariant derivative of a spinor is defined according to

$$
\nabla_{M} \epsilon=\partial_{M} \varepsilon+\frac{1}{4} \Omega^{A B}{ }_{M} \Gamma_{A B} \varepsilon
$$


where $\Omega$ is the spin connection. Note that the gravitino variation can then be written in the form

$$
\delta \Psi_{M}=\partial_{M} \varepsilon+\frac{1}{4} \Omega_{-}^{A B}{ }_{M} \Gamma_{A B} \varepsilon
$$

where

$$
\Omega_{ \pm M}^{A B}=\Omega_{M}^{A B} \pm \frac{1}{2} H_{M}^{A B}
$$

Explicitly the components of the spin connection are

$$
\begin{aligned}
\Omega_{ \pm a}^{w_{1}}= & \frac{1}{2} e^{2 A}\left(H_{w_{1}} \mp \star_{b} H_{w_{1}}\right)_{a b} e^{b}, \\
\Omega_{ \pm a}^{w_{2}}= & \frac{1}{2} e^{2 A}\left(H_{w_{2}} \mp \star_{b} H_{w_{2}}\right)_{a b} e^{b} \\
\Omega_{ \pm b}^{a}= & 2\left[\partial^{a} A e_{b}-\partial_{b} A e^{a} \mp\left(\star_{b} d A\right)^{a}{ }_{b c} e^{c}\right]+\omega^{a}{ }_{b} \\
& -\frac{1}{2} e^{4 A}\left(H_{w_{1}} \pm \star_{b} H_{w_{1}}\right)^{a}{ }_{b} E^{w_{1}}-\frac{1}{2} e^{4 A}\left(H_{w_{2}} \pm \star_{b} H_{w_{2}}\right)^{a}{ }_{b} E^{w_{2}} .
\end{aligned}
$$

Note the sign differences between the first two components of the spin connection and the last one. These sign differences will play a crucial role in the supersymmetry analysis.

Under the decomposition $S O(9,1) \rightarrow S O(3,1) \times S O(6)$ a $10 d$ Weyl spinor decomposes as $\mathbf{1 6} \rightarrow(\mathbf{2}, \mathbf{4})+\left(\mathbf{2}^{\prime}, \mathbf{4}^{\prime}\right)$. Imposing the Majorana condition we set

$$
\epsilon=\zeta \otimes \eta+\zeta^{\star} \otimes \eta^{\star}
$$

where $\zeta \otimes \eta$ transforms as $(\mathbf{2}, \mathbf{4})$. Since the complex conjugate is not an independent spinor each 6d Weyl spinor gives rise to one minimal 4d supersymmetry.

Lets solve the supersymmetry constraints. The gravitino condition with the index in the external space-time is satisfied if the spinor does not depend on the coordinates of the external space-time. Projecting onto spinors with definite $4 \mathrm{~d}$ chirality the supersymmetry conditions become

$$
\begin{aligned}
& \nabla_{M} \eta-\frac{1}{4} \mathcal{H}_{M} \eta=0, \\
& \not \phi_{\mathrm{h}} \eta-\frac{1}{2} \mathcal{H} \eta=0, \\
& \mathcal{F} \eta=0,
\end{aligned}
$$

which are equations constraining the $6 \mathrm{~d}$ spinor $\eta$. To solve this supersymmetry conditions the spinor $\eta$ has to satisfy

$$
\partial_{w_{i}} \eta=0 \quad \text { and } \quad \partial_{i} \eta+\frac{1}{4} \omega^{a b}{ }_{i} \gamma_{a b} \eta=0
$$


i.e. $\eta$ is a covariantly constant spinor on the base. We denote the two covariantly constant spinors of $\mathrm{K} 3$ by $\eta_{k}, k=1,2$. Moreover, we require $\eta_{k}$ to solve

$$
\begin{aligned}
& \left(H_{w_{1}}-\star_{b} H_{w_{1}}\right)_{a b} \gamma^{a b} \eta_{k}=\left(H_{w_{2}}-\star_{b} H_{w_{2}}\right)_{a b} \gamma^{a b} \eta_{k}=0, \\
& \left(H_{w_{1}}+\star_{b} H_{w_{1}}\right)_{a b} \gamma^{w_{1} a} \eta_{k}+\left(H_{w_{2}}+\star_{b} H_{w_{2}}\right)_{a b} \gamma^{w_{2} a} \eta_{k}=0,
\end{aligned}
$$

which after introducing complex coordinates $w=w_{1}+i w_{2}$, so that

$$
H_{w}=\frac{1}{2}\left(H_{w_{1}}-i H_{w_{2}}\right) \quad \text { and } \quad H_{\bar{w}}=\frac{1}{2}\left(H_{w_{1}}+i H_{w_{2}}\right)
$$

take the form

$$
\begin{aligned}
& {\left[\left(1-\star_{b}\right) H_{w}\right]_{a b} \gamma^{a b} \eta_{k}=0,} \\
& {\left[\left(1-\star_{b}\right) H_{\bar{w}}\right]_{a b} \gamma^{a b} \eta_{k}=0,} \\
& {\left[\left(1+\star_{b}\right) H_{w}\right]_{a b} \gamma^{w a} \eta_{k}+\left[\left(1+\star_{b}\right) H_{\bar{w}}\right]_{a b} \gamma^{\bar{w} a} \eta_{k}=0 .}
\end{aligned}
$$

Note that the contributions involving the warp factor arising from the spin connection components $\Omega_{ \pm}^{a b}$ and contributing to the component of the gravitino variation along the base cancel since the two spinors $\eta_{k}$ have positive chirality on the base i.e.

$$
-\gamma_{1234} \eta_{k}=\eta_{k} \quad k=1,2
$$

Now depending on the choice of flux different amounts of supersymmetry are preserved [6]. The different cases are

$\triangleright$ if $H_{w}$ is proportional to an anti-self dual $(1,1)$ form on the K3 base, the conditions (3.29) are satisfied for both spinors $\eta_{k}, k=1,2$. An $\mathrm{N}=2$ supersymmetry is preserved in $4 \mathrm{~d}$. Indeed, the third condition is trivially satisfied and the first two conditions are satisfied since the anti-self dual $(1,1)$ forms are primitive with respect to the base.

$\triangleright$ if $H_{w}$ is proportional to the self-dual $(0,2)$ form on the base the supersymmetry generated by $\eta_{1}$ is preserved while $\eta_{2}=0$. There is an $\mathrm{N}=1$ unbroken supersymmetry in $4 \mathrm{~d}$.

$\triangleright$ if $H_{w}$ is proportional to the self-dual $(2,0)$ form on the base the supersymmetry generated by $\eta_{2}$ is unbroken while $\eta_{1}=0$. There is an $N=1$ ' unbroken supersymmetry in $4 \mathrm{~d}$.

$\triangleright$ if $H_{w}$ is proportional to the self-dual $(1,1)$ form on the base (3.29) requires the two spinors to vanish. So $\mathrm{N}=0$ in $4 \mathrm{~d}$. 


\section{The $\alpha^{\prime}$ corrected torsional heterotic geometry}

In this section we will consider $\alpha^{\prime}$ corrections to the torsional heterotic geometries. We

will see that these $\alpha^{\prime}$ corrections to the background are required since otherwise the $\alpha^{\prime}$ corrected equations of motion are not satisfied. Once the background is corrected in $\alpha^{\prime}$ compact solutions become possible. As a first step to solve the Bianchi identity we need to compute $\operatorname{tr}\left(R_{+} \wedge R_{+}\right)$, which appears on the right hand side of the Bianchi identity.

\section{1 $\operatorname{tr}\left(R_{+} \wedge R_{+}\right)$}

In general, the curvature two-form is defined by

$$
R_{B}^{A}=d \Omega_{B}^{A}+\Omega_{C}^{A} \wedge \Omega_{B}^{C},
$$

for some connection $\Omega$. According to Bergshoeff and de Roo [21] the connection used in the supersymmtry transformations is $\Omega_{-}$while in the Bianchi identity the $\Omega_{+}$connection is used. The connection coefficients are

$$
\begin{aligned}
& \Omega_{+a}^{w_{k}}=\frac{1}{2} e^{2 A}\left(H_{w_{k}}-\star_{b} H_{w_{k}}\right)_{i j} e_{a}^{i} d y^{j}, \quad k=1,2 \\
& \Omega_{+_{b}}^{a}=\sigma_{b}^{a}+\omega_{b}^{a}-\frac{1}{2}\left(H_{w_{k}}+\star_{b} H_{w_{k}}\right)_{i j} E_{c}^{i} E_{b}^{j} \eta^{a c} E^{w_{k}},
\end{aligned}
$$

where, the last term involves a sum over $k=1,2$. We denote with $E^{a}$ the vielbeine of the warped base while $e^{a}$ are those of the unwarped K3. Moreover,

$$
\sigma_{a b}=2\left[\partial_{a} A e_{b}-\partial_{b} A e_{a}-\left(\star_{b} d A\right)_{a b c} e^{c}\right] .
$$

Note that $\sigma_{a b}$ is self-dual in its indices, i.e. it satisfies

$$
\sigma_{a b}=\frac{1}{2} \varepsilon_{a b c d} \sigma^{c d}
$$

We are denoting the spin connection coefficients and curvature two-form of the K3 base by $\omega^{a}{ }_{b}$ and $r_{b}^{a}$.

Before describing in detail the results for the curvature two-form and $\operatorname{tr}\left(R_{+} \wedge R_{+}\right)$, where $R_{+}$is computed with respect to the $\Omega_{+}$connection, we will first establish that the curvature two-form of the torsional space is of type $(1,1)$ to leading order in $\alpha^{\prime}$ if computed with respect to the $\Omega_{+}$connection. This implies that $\operatorname{tr}\left(R_{+} \wedge R_{+}\right)$is a $(2,2)$ form which is a necessary condition for the Bianchi identity to admit a nontrivial solution. Indeed, up to terms of $O\left(\alpha^{\prime 2}\right)$ unbroken supersymmetry requires the 
flux and the fundamental $(1,1)$ form to be related according to $\mathcal{H}=i(\partial-\bar{\partial}) J$. As a result $d \mathcal{H}=-2 i \partial \bar{\partial} J$ is a $(2,2)$ form. This is the left hand side of the Bianchi identity. The right hand side of the Bianchi identity is $\operatorname{tr}\left(R_{+} \wedge R_{+}\right)$, which is required to be a four-form of type $(2,2)$ since otherwise the background is over-constrained.

Here we follow the presentation of ref. [29]. By definition

$$
\Omega_{+M}^{A B}=\Omega_{M}^{A B}+\frac{1}{2} H_{M}^{A B}
$$

which implies that the connection in the coordinate basis is modified to

$$
\Gamma_{+I K}^{J}=G^{J L}\left(E_{L}^{A} \partial_{I} E_{K}^{A}+\Omega_{+}^{A B}{ }_{I} E_{L}^{A} E_{K}^{B}\right)=\Gamma_{I K}^{J}-\frac{1}{2} \mathcal{H}_{I K}{ }^{J}
$$

By definition

$$
\Gamma_{I K}^{J}=\frac{1}{2} g^{J N}\left(\partial_{I} g_{N K}+\partial_{K} g_{N I}-\partial_{N} g_{I K}\right)
$$

Supersymmtry requires $\mathcal{H}$ to be related to the derivative of the metric according to

$$
\mathcal{H}_{M N \bar{P}}=-\partial_{M} g_{N \bar{P}}+\partial_{N} g_{M \bar{P}}
$$

and the complex conjugate. Here we have introduced complex coordinates. Using the fact that the metric of the torsional space is hermitian eqn. (4.7) implies that the non-vanishing connection coefficients are

$$
\Gamma_{+J K}^{I}=g^{I \bar{N}} \partial_{J} g_{K \bar{N}} \quad \text { and } \quad \Gamma_{+J \bar{K}}^{I}=g^{I \bar{N}} \partial_{\bar{K}} g_{J \bar{N}}-g^{I \bar{N}} \partial_{\bar{N}} g_{J \bar{K}}
$$

So in contrast to Kähler geometry there are connection coefficients with mixed indices.

The Riemann tensor is obtained from the connection coefficients according to

$$
R_{M N}{ }_{L}^{K}=\partial_{M} \Gamma_{N L}^{K}-\partial_{N} \Gamma_{M L}^{K}+\Gamma_{M R}^{K} \Gamma_{N L}^{R}-\Gamma_{N R}^{K} \Gamma_{M L}^{R}
$$

and the curvature two-form is related to the Riemann tensor according to

$$
R_{B}^{A}=\frac{1}{2} R_{C D}{ }_{B}^{A} E^{C} E^{D} .
$$

Introducing complex coordinates it is not difficult to see that

$$
R_{+M N}{ }_{L}^{K}=R_{+M N}{ }_{L}^{\bar{K}}=R_{+M N}{ }^{\bar{K}}{ }_{\bar{L}}=0
$$

Moreover,

$$
R_{+\bar{M} \bar{N}}^{\bar{K}}{ }_{L}=g^{P \bar{K}}\left(g_{P[\bar{N}, \bar{M}] L}-g_{L[\bar{N}, \bar{M}] P}\right)=O\left(\alpha^{\prime}\right)
$$


This quantity is subleading since the right hand side is the $(2,2)$ component of $d \mathcal{H}$ which is $O\left(\alpha^{\prime}\right)$ after using the Bianchi identity. Therefore we conclude that to leading order in $\alpha^{\prime}, \operatorname{tr}\left(R_{+} \wedge R_{+}\right)$is of type $(2,2)$.

Next we present the explicit results for the curvature two-forms and $\operatorname{tr}\left(R_{+} \wedge R_{+}\right)$ and show how to solve the Bianchi identity. We will focus on solutions with $\mathrm{N}=2$ supersymmetry.

\section{$4.2 \quad \mathrm{~N}=2$ background at $O\left(\alpha^{\prime}\right)$}

In this case the forms $H_{w_{i}}$ are proportional to anti-self dual $(1,1)$ forms on the K3 base. From (4.2) we see that the only non-vanishing components of the spin connection are

$$
\begin{aligned}
& \Omega_{+a}^{w_{k}}=e^{2 A}\left(H_{w_{k}}\right)_{i j} e_{a}^{i} d y^{j}, \quad k=1,2 \\
& \Omega_{+b}^{a}=\sigma_{b}^{a}+\omega_{b}^{a}{ }_{b} .
\end{aligned}
$$

In this case the curvature two-form computed with respect to the $\Omega_{+}$connection is a two-from on K3 explicitly given by

$$
\begin{aligned}
R_{w_{2}}^{w_{1}} & =-e^{4 A}\left(H_{w_{1}}\right)_{a}\left(H_{w_{2}}\right)^{a} \\
R_{w_{k}}^{a} & =-\nabla\left[e^{2 A}\left(H_{w_{k}}\right)_{a}\right]-e^{2 A}\left(H_{w_{k}}\right)_{b} \sigma^{b}{ }_{a}, \quad k=1,2 \\
R_{b}^{a} & =r^{a}{ }_{b}+\nabla \sigma^{a}{ }_{c}+\sigma^{a}{ }_{c} \sigma^{c}{ }_{b}-e^{4 A}\left(H_{w_{k}}\right)_{a}\left(H_{w_{k}}\right)_{b},
\end{aligned}
$$

where $r_{b}^{a}$ is the curvature two-form of $\mathrm{K} 3$ and $\nabla$ is the covariant derivative with respect to the $\omega^{a}{ }_{b}$ connection. Explicitly

$$
\nabla \sigma_{b}^{a}=d \sigma_{b}^{a}+\omega^{a}{ }_{c} \sigma_{b}^{c}+\sigma_{c}^{a} \omega_{b}^{c} \text {. }
$$

A convenient way to compute $\operatorname{tr}\left(R_{+} \wedge R_{+}\right)$is to use the Chern-Simons formula which relates the results for $\operatorname{tr}(R \wedge R)$ computed with two connections $\Gamma$ and $\tilde{\Gamma}$ according to

$$
\operatorname{tr}(R \wedge R)-\operatorname{tr}(\tilde{R} \wedge \tilde{R})=d Q(\Gamma, \tilde{\Gamma})
$$

where

$$
Q(\Gamma, \tilde{\Gamma})=2 \alpha \wedge R-\alpha \wedge d \alpha-2 \alpha \wedge \Gamma \wedge \alpha+\frac{2}{3} \alpha \wedge \alpha \wedge \alpha
$$

where $\alpha=\Gamma-\tilde{\Gamma}$. Setting

$$
\begin{aligned}
\tilde{\Gamma}_{b}^{a} & =\Omega_{+b}^{a} & \text { and } & \tilde{\Gamma}_{a}^{w_{k}}=\Omega_{+}{ }^{w_{k}}{ }_{a} \\
\Gamma_{b}^{a}{ }_{b}=\Omega_{+b}^{a} & \text { and } & \Gamma^{w_{k}}{ }_{a}=0, & k=1,2,
\end{aligned}
$$


or in other words choosing

$$
\alpha_{b}^{a}=0 \quad \text { and } \quad \alpha^{w_{k}}{ }_{a}=-e^{2 A}\left(H_{w_{k}}\right)_{i j} e_{a}^{i} d y^{j}
$$

we obtain

$\operatorname{tr}\left(R_{+} \wedge R_{+}\right)=\operatorname{tr}[R(\Gamma) \wedge R(\Gamma)]+2 d\left\{e^{2 A}\left(H_{w_{k}}\right)_{b} \nabla\left[e^{2 A}\left(H_{w_{k}}\right)_{b}\right]+e^{4 A}\left(H_{w_{k}}\right)_{b} \sigma^{b}{ }_{c}\left(H_{w_{k}}\right)_{c}\right\}$

where

$$
\operatorname{tr}[R(\Gamma) \wedge R(\Gamma)]=-\left(\nabla \sigma_{b}^{a}+r_{b}^{a}+\sigma_{c}^{a} \sigma_{b}^{c}\right)\left(\nabla \sigma_{a}^{b}+r_{a}^{b}+\sigma_{c}^{b} \sigma_{a}^{c}\right)
$$

This result can be further simplified by using the Chern-Simons formula again, this time with

$$
\tilde{\Gamma}_{b}^{a}=\omega_{b}^{a} \quad \text { and } \quad \Gamma_{b}^{a}=\omega_{b}^{a}+\sigma_{b}^{a} .
$$

The result is

$$
\operatorname{tr}[R(\Gamma) \wedge R(\Gamma)]=\operatorname{tr}(r \wedge r)-2^{4} d\left[2\left(\nabla^{2} A\right) \star d A-\star d(\nabla A)^{2}-8(\nabla A)^{2} \star d A\right]
$$

A straightforward but tedious computation then shows

$$
\begin{aligned}
\operatorname{tr}\left(R_{+} \wedge R_{+}\right)= & \operatorname{tr}(r \wedge r)+4 d \star_{b} d\left(\nabla^{2} A\right)+ \\
& d \star_{b} d\left[\left(\nabla^{2} e^{-4 A}+|H|^{2}\right) e^{4 A}\right]+ \\
& 2 d\left[\left(\nabla^{2} e^{-4 A}+|H|^{2}\right) \star_{b} d e^{4 A}\right],
\end{aligned}
$$

where

$$
|H|^{2}=\left|H_{w_{1}}\right|^{2}+\left|H_{w_{2}}\right|^{2} \text {. }
$$

Note that the last two lines in eqn. (4.25) involve the leading order equation of motion (3.17). Thus we establish that for solutions preserving an $\mathrm{N}=2$ supersymmetry in four dimensions $\operatorname{tr}\left(R_{+} \wedge R_{+}\right)$is a $(2,2)$ form with components along the K3 base only. Note that this fact is a consequence of having used the $\Omega_{+}$connection to compute $\operatorname{tr}\left(R_{+} \wedge R_{+}\right)$. Since $\operatorname{tr}\left(R_{+} \wedge R_{+}\right)$has components along the base only the fiber is not required to be of $O\left(\alpha^{\prime}\right)$ and can be chosen to be large.

Next we will use this result and solve the Bianchi identity

$$
d \mathcal{H}=\frac{\alpha^{\prime}}{4}[\operatorname{tr}(R \wedge R)-\operatorname{tr}(\mathcal{F} \wedge \mathcal{F})]
$$

to $O\left(\alpha^{\prime}\right)$. 
First we note that the second and third line on the right hand side of Eq.(4.25) are proportional to the dual of $d \mathcal{H}$ and are therefore $O\left(\alpha^{\prime}\right)$. As a result they contribute to the Bianchi identity only to $O\left(\alpha^{\prime 2}\right)$. Keeping all terms up to $O\left(\alpha^{\prime}\right)$ the Bianchi identity becomes

$$
d \star_{b} d e^{-4 A}-\star_{b} H_{w_{k}} \wedge H_{w_{k}}+O\left(\alpha^{\prime}\right)=\frac{\alpha^{\prime}}{4}[\operatorname{tr}(r \wedge r)-\operatorname{tr}(\mathcal{F} \wedge \mathcal{F})]+\alpha^{\prime} d \star_{b} d\left(\nabla^{2} A\right) .
$$

Here we have allowed a correction to $O\left(\alpha^{\prime}\right)$ on the left hand side. Since the supersymmtry transformations receive only corrections at $O\left(\alpha^{\prime 2}\right)$ any corrections to the left hand side of eqn.(4.28) have to solve the leading order supersymmetry conditions. Since the supersymmtry conditions do not determine $A(y)$ we can redefine the warp factor and still obtain a supersymmetric situation. In particular if we define

$$
e^{-4 A^{\prime}}=e^{-4 A}+\alpha^{\prime} \nabla^{2} A .
$$

and allow the background to receive an $O\left(\alpha^{\prime}\right)$ correction according to

$$
\begin{aligned}
& \phi_{\mathrm{h}}=-2 A^{\prime}(y), \\
& \mathcal{H}=\star_{b} d e^{-4 A^{\prime}(y)}-\star_{b} H_{w_{1}} \wedge E_{w_{1}}-\star_{b} H_{w_{2}} \wedge E_{w_{2}} \\
& d s_{\text {het }}^{2}=\eta_{\mu \nu} d x^{\mu} d x^{\nu}+e^{-4 A^{\prime}(y)} g_{i j} d y^{i} d y^{j}+E_{w_{1}} E_{w_{1}}+E_{w_{2}} E_{w_{2}}
\end{aligned}
$$

supersymmtry will still be preserved. To this order in $\alpha^{\prime}$ the Bianchi identity becomes an equation of Laplace type, namely

$$
d \star_{b} d e^{-4 A}-\star_{b} H_{w_{k}} \wedge H_{w_{k}}=\frac{\alpha^{\prime}}{4}[\operatorname{tr}(r \wedge r)-\operatorname{tr}(\mathcal{F} \wedge \mathcal{F})]
$$

Note that we have obtained a linear differential for the dilaton even though the Bianchi identity could, in principle, lead to a highly non-linear differential equation. This fact depends crucially on choosing the $\Omega_{+}$connection to construct $\operatorname{tr}\left(R_{+} \wedge R_{+}\right)$. There is a preferred set of fields for which this connection is required by space-time supersymmetry as shown by Bergshoeff and de Roo [21]. A different choice of connection is always possible but it leads to a different choice of fields for which in general the supersymmetry transformations will receive corrections at $O\left(\alpha^{\prime}\right)$. We have found a differential equation of Laplace type using the $\Omega_{+}$connection and the solvability of the equation is immediate if the integrated equation is satified. Choosing the hermitian connection, on the other hand, will lead to a highly non-linear differential equation of Monge-Ampere type as shown in refs. [25] [26] . 
In the following we will show that the $\alpha^{\prime}$ corrected background solves the equations of motion presented in section 3.2. First we note that the equation of motion of $\mathcal{B}$ is satisfied since in the background (4.30)

$$
\star_{10} \mathcal{H}=-e^{2 \phi_{\mathrm{h}}} d\left(e^{-2 \phi_{\mathrm{h}}} E^{w_{1}} \wedge E^{w_{2}}\right) \wedge d x^{0123} .
$$

The Bianchi identity for $\mathcal{H}$ is solved by construction.

To solve the equations of motion for the metric we first establish some properties of the Riemann tensor. First, the Ricci tensor of the torsional metric is

$$
R_{i j}=4 \nabla_{i} \partial_{j} A^{\prime}+8 \partial_{i} A^{\prime} \partial_{j} A^{\prime}-\frac{1}{2} e^{4 A^{\prime}} H_{w_{k} a i} H_{j}^{w_{k} a}+g_{i j}\left[2 \nabla^{2} A^{\prime}-8\left(\partial A^{\prime}\right)^{2}\right],
$$

where $(i, j)$ are indices on the base and $\nabla_{i}$ involves connections on the base only. Note that this derivative is not identical to $\nabla_{i}^{(6)}$, which is the covariant derivative constructed with respect to the connections on the six-dimensional torsional space. So for example

$$
\nabla_{i}^{(6)} \partial_{j} \phi_{\mathrm{h}}=\nabla_{i} \partial_{j} \phi_{\mathrm{h}}-8 \partial_{i} A^{\prime} \partial_{j} A^{\prime}+4 g_{i j}\left(\partial A^{\prime}\right)^{2}
$$

Up to terms of $O\left(\alpha^{\prime}\right)$ the curvature two-form constructed from the $\Omega_{+}$connection $R_{+B}^{A}$ satisfies

$$
\star_{b} R_{+}{ }_{B}^{A}=-R_{+}{ }^{A}{ }_{B}+O\left(\alpha^{\prime}\right)
$$

This condition can be derived using the integrability condition of the supersymmetry constrain on the gravitino

$$
\left[\nabla_{-M}, \nabla_{-N}\right] \varepsilon=\frac{1}{4} R_{-M N P Q} \Gamma^{P Q} \varepsilon=0,
$$

which implies

$$
R_{-M N P Q} J^{P Q}=0
$$

Moreover, one has

$$
R_{-P Q M N}=R_{+M N P Q}-2 \nabla_{[P} \mathcal{H}_{M N Q]}=R_{+M N P Q}+O\left(\alpha^{\prime}\right),
$$

which implies

$$
R_{+P Q M N} J^{P Q}+O\left(\alpha^{\prime}\right)=0 .
$$

From here we obtain the following identity

$$
R_{+m P A B} R_{+n}^{P A B}=\frac{1}{4} R_{+P Q A B} R_{+}{ }^{P Q A B} g_{m n}+O\left(\alpha^{\prime}\right)
$$


where now $(m, n)$ are indices on the K3 base only, while if these indices are along the fiber the result vanishes. Also,

$$
\operatorname{tr}\left(R_{+} \wedge R_{+}\right)=-\frac{1}{2} R_{+P Q A B} R_{+}^{P Q A B} \star_{b} 1+O\left(\alpha^{\prime}\right) .
$$

Using the above result for the curvature we can now verify the equation of motion for the metric and the dilaton. The only non-trivial component of the Einstein equation is the $(M, N)=(m, n)$ component with both indices along the base. All terms, except the ones proportional to the base metric $g_{m n}$ cancel. The coefficient of $g_{m n}$, on the other hand, turns out to be the Hodge dual of the Bianchi identity (4.31), as can be verified with a bit of patience. As a result the Einstein equation, Bianchi identity and equation of motion for $\mathcal{B}$ are satisfied. Explicit computation shows that also the dilaton equation of motion is solved.

We end by describing torsional spaces with an $N=2$ supersymmetry in which the twist of the fiber is 'exchanged' by vacuum expectation values of abelian gauge fields. This type of solutions were suggested in refs. [27] [28]. In this case the torus fiber is not twisted and the background fields are

$$
\begin{aligned}
d s^{2} & =\eta_{\mu \nu} d x^{\mu} d x^{\nu}+e^{-4 A^{\prime}(y)} g_{i j} d y^{i} d y^{j}+d w_{1}^{2}+d w_{2}^{2}, \\
\mathcal{H} & =\star_{b} d e^{-4 A^{\prime}(y)} \\
\mathcal{F} & =\mathcal{F}_{i \bar{j}} d y^{i} d y^{\bar{j}} \\
\phi_{\mathrm{h}} & =-2 A^{\prime}(y)
\end{aligned}
$$

where now an abelian gauge field is included as part of the background and $\mathcal{F}$ is an anti-self dual form on K3. This background solves the supersymmetry constraints preserving an $\mathrm{N}=2$ supersymmtry. Moreover, it is not difficult to see that the Bianchi identity reduces to the differential equation

$$
\begin{aligned}
-\nabla^{2} e^{-4 A(y)} \star_{b} 1= & \frac{\alpha^{\prime}}{4}[\operatorname{tr}(r \wedge r)-\operatorname{tr}(\mathcal{F} \wedge \mathcal{F})] \\
& +\frac{3 \alpha^{\prime}}{4} d\left(\nabla^{2} e^{-4 A} \star_{b} d e^{4 A}\right) \\
& +\frac{\alpha^{\prime}}{4} d\left(e^{4 A} \star_{b} d \nabla^{2} e^{-4 A}\right) .
\end{aligned}
$$

The computation of $\operatorname{tr}\left(R_{+} \wedge R_{+}\right)$for these solutions is greatly simplified since the fiber is not twisted. In this case the second and third lines on the right hand side of eqn. (4.43) are again corrections of order $O\left(\alpha^{\prime 2}\right)$ or higher and can only be consistently 
taken into account once the supersymmetry transformations are corrected to $O\left(\alpha^{\prime 2}\right)$. Therefore to $O\left(\alpha^{\prime}\right)$ the differential equation is again of Laplace type and solvability is guaranteed. The form of the $O\left(\alpha^{\prime 2}\right)$ corrections to the supersymmetry transformations has been described in ref. [21]. The analysis to $O\left(\alpha^{\prime 2}\right)$ of solutions preserving an $\mathrm{N}=2$ supersymmetry and the solvability of the Bianchi identity for backgrounds preserving an $\mathrm{N}=1$ supersymmetry is currently under investigation [30].

\section{Acknowledgements}

It is our pleasure to thank Aaron Bergman, Greg Moore and Savdeep Sethi for helpful discussions. K. B. and G. G. would like to thank the KITP for hospitality during the final stages of this work.

This work is supported in part by NSF Grant No. PHY-0505757 and by NSF Grant No. PHY-05-51164 and by the University of Texas A\&M.

\section{A Conventions}

In this appendix, we summarize our conventions and quote some useful formulas.

We use indices

$$
M, N, \ldots \mu, \nu, \ldots, i, j, \ldots, w_{1}, w_{2}, \quad\left(A, B, \ldots \alpha, \beta, \ldots, a, b, \ldots, w_{1}, w_{2}\right)
$$

to denote the coordinate (non-coordinate) bases of any six-dimensional space, of fourdimensional Minkowski space-time, of the base and of the fiber, respectively. For coordinates on the four-dimensional base of the six-dimensional space, we use $y^{i}$ while we denote the fiber coordinates by $w_{i}, i=1,2$.

We define the chirality operators

$$
\Gamma^{(4)}=-i \Gamma^{0} \Gamma^{1} \Gamma^{2} \Gamma^{3}, \quad \Gamma^{\left(4^{\prime}\right)}=-\Gamma^{4} \Gamma^{5} \Gamma^{6} \Gamma^{7}, \quad \Gamma_{\star}=-i \Gamma^{8} \Gamma^{9}
$$

where $\Gamma^{(4)}, \Gamma^{\left(4^{\prime}\right)}$, and $\Gamma_{\star}$ are the chirality operators for external space, K3 base and the $T^{2}$ fibre, from which we get

$$
\Gamma^{(10)}=\Gamma^{(4)} \Gamma^{\left(4^{\prime}\right)} \Gamma_{\star}=\Gamma^{0} \cdots \Gamma^{9}
$$

for the $10 \mathrm{~d}$ space. In type the IIB theory, the $10 \mathrm{~d}$ spinor $\varepsilon$ satisfies

$$
\Gamma^{(10)} \varepsilon=-\varepsilon
$$


We also choose the orientation

$$
\epsilon^{4567 w_{1} w_{2}}=-1
$$

The Riemann tensor is defined by

$$
R_{\mu \nu}{ }_{B}^{A}=\partial_{\mu} \Omega_{B \nu}^{A}-\partial_{\nu} \Omega_{B \mu}^{A}+\Omega_{C \mu}^{A} \Omega_{B \nu}^{C}-\Omega_{C \nu}^{A} \Omega_{B \mu}^{C}
$$

and

$$
\operatorname{trR} \wedge \mathrm{R}=R_{B}^{A} \wedge R_{B}^{A} .
$$

We use the notation

$$
H_{w_{i}}=\frac{1}{2} H_{w_{i} a b} e^{a} \wedge e^{b}, \quad\left(H_{w_{i}}\right)_{a}=H_{w_{i} a b} e^{b}, \quad H_{w_{i} a b}=H_{w_{i} m n} e_{a}^{m} e_{b}^{n}
$$

and

$$
|H|^{2}=\frac{1}{2} H_{w_{1} a b} H_{w_{1}}{ }^{a b}+\frac{1}{2} H_{w_{2} a b} H_{w_{2}}{ }^{a b}
$$

with $e^{a}$ the vielbein for unwarped K3 base.

To compute the $\operatorname{tr} R \wedge R$, it is convenient to use the following results

$$
\begin{array}{lc}
A_{i j} A^{i}{ }_{k}=\frac{1}{4} A_{m n} A^{m n} g_{j k}, & S_{i j} S^{i}{ }_{k}=\frac{1}{4} S_{m n} S^{m n} g_{j k} \\
A_{i j} S^{i}{ }_{k}=A_{i k} S^{i}{ }_{j}, & A_{i j} S^{i j}=0 \\
A_{i j}=-\frac{1}{2} \epsilon_{i j}{ }^{k l} A_{k l}, & S_{i j}=\frac{1}{2} \epsilon_{i j}{ }^{k l} S_{k l}
\end{array}
$$

where $A_{i j}$ are the components of any anti-self-dual two form on the K3 base, and $S_{i j}$ are the components of any self-dual two form on the K3 base.

\section{References}

[1] C. M. Hull and E. Witten, "Supersymmetric sigma models and the heterotic string," Phys. Lett. B 160, 398 (1985).

[2] A. Strominger. "Superstrings with torsion," Nucl. Phys. B274 (1986) 253-284.

[3] K. Becker and S. Sethi, "Torsional heterotic geometries", 0903.3769.

[4] K. Dasgupta, G. Rajesh, and S. Sethi, "M theory, orientifolds and G-flux", JHEP 9908023 (1999), hep-th/9908088. 
[5] K. Becker and K. Dasgupta, "Heterotic strings with torsion," JHEP 0211, 006 (2002), hep-th/0209077.

[6] K. Becker, M. Becker, J. X. Fu, L. S. Tseng and S. T. Yau, "Anomaly cancellation and smooth non-Kaehler solutions in heterotic string theory," Nucl. Phys. B 751, 108 (2006), hep-th/0604137.

[7] P. K. Tripathy and S. P. Trivedi, "Compactification with flux on K3 and tori", JHEP 0303, 028 (2003), hep-th/0301139.

[8] G. W. Moore, "Les Houches lectures on strings and arithmetic", hep-th/0401049.

[9] A. Sen, "F-theory and orientifolds," Nucl. Phys. B 475, 562 (1996), hepth/9605150.

[10] A. Sen, "Orientifold limit of F-theory vacua," Phys. Rev. D 55, 7345 (1997), hep-th/9702165.

[11] M. B. Green, J. A. Harvey and G. W. Moore, "I-brane inflow and anomalous couplings on D-branes," Class. Quant. Grav. 14, 47 (1997), hep-th/9605033.

[12] J. F. Morales, C. A. Scrucca and M. Serone, "Anomalous couplings for D-branes and O-planes," Nucl. Phys. B 552, 291 (1999), hep-th/9812071.

[13] C. A. Scrucca and M. Serone, "Anomalies and inflow on D-branes and O-planes," Nucl. Phys. B 556, 197 (1999), hep-th/9903145.

[14] C. A. Scrucca and M. Serone, "A note on the torsion dependence of D-brane RR couplings," Phys. Lett. B 504, 47 (2001), hep-th/0010022.

[15] B. Craps and F. Roose, "Anomalous D-brane and orientifold couplings from the boundary state," Phys. Lett. B 445, 150 (1998), hep-th/9808074.

[16] B. J. Stefanski, Nucl. Phys. B 548, 275 (1999), hep-th/9812088.

[17] B. Craps and F. Roose, "(Non-)anomalous D-brane and O-plane couplings: The normal bundle," Phys. Lett. B 450, 358 (1999), hep-th/9812149.

[18] C. G. . Callan, E. J. Martinec, M. J. Perry and D. Friedan, "Strings in background fields," Nucl. Phys. B 262, 593 (1985). 
[19] R. R. Metsaev and A. A. Tseytlin, "Order $\alpha^{\prime}$ (two Loop) equivalence of the string equations of motion and the sigma model Weyl invariance conditions: dependence on the dilaton and the antisymmetric tensor," Nucl. Phys. B293 (1987) 385.

[20] E. Bergshoeff and M. de Roo, "Supersymmetric Chern-Simons terms in ten dimensions," Phys. Lett. B218 (1989) 210.

[21] E. A. Bergshoeff and M. de Roo, "The quartic effective action of the heterotic string and supersymmetry," Nucl. Phys. B328 (1989) 439.

[22] W. A. Chemissany, M. de Roo, and S. Panda, " $\alpha$-Corrections to heterotic superstring effective action revisited," JHEP 08 (2007) 037, 0706.3636.

[23] S. S. Gubser, "Supersymmetry and F-theory realization of the deformed conifold with three-form flux,", hep-th/0010010.

[24] M. Grana and J. Polchinski, "Supersymmetric three-form flux perturbations on AdS(5)," Phys. Rev. D 63, 026001 (2001), hep-th/0009211.

[25] J. X. Fu and S. T. Yau, "Existence of supersymmetric Hermitian metrics with torsion on non-Kaehler manifolds", hep-th/0509028.

[26] J. X. Fu and S. T. Yau, "The theory of superstring with flux on non-Kähler manifolds and the complex Monge-Ampere equation", hep-th/0604063.

[27] M. Becker, L. Tseng, and S.-T. Yau, "Heterotic Kähler/non-Kähler Transitions", 0706.4290 .

[28] S. Sethi, "A Note on heterotic dualities via M-theory," Phys. Lett. B 659, 385 (2008), 0707.0295.

[29] A. Sen, "(2, 0) supersymmetry and space-time supersymmetry in the heterotic string theory," Nucl. Phys. B 278, 289 (1986).

[30] K. Becker and G. Guo, under investigation. 